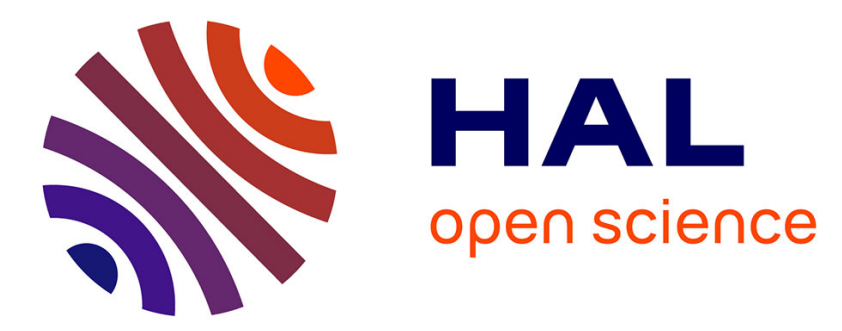

\title{
Stabilization of a class of switched dynamic systems: the Riccati-equation-based Approach
}

M Bonilla, N A Aguillón,, M A Ortiz Castillo, Jean Jacques Loiseau, Michel Malabre, V Azhmyakov, S Salazar

\section{- To cite this version:}

M Bonilla, N A Aguillón,, M A Ortiz Castillo, Jean Jacques Loiseau, Michel Malabre, et al.. Stabilization of a class of switched dynamic systems: the Riccati-equation-based Approach. IMA Journal of Mathematical Control and Information, 2022, 39 (2), pp.518-532. 10.1093/imamci/dnab022 . hal03321806

\section{HAL Id: hal-03321806 \\ https://hal.science/hal-03321806}

Submitted on 27 Oct 2021

HAL is a multi-disciplinary open access archive for the deposit and dissemination of scientific research documents, whether they are published or not. The documents may come from teaching and research institutions in France or abroad, or from public or private research centers.
L'archive ouverte pluridisciplinaire HAL, est destinée au dépôt et à la diffusion de documents scientifiques de niveau recherche, publiés ou non, émanant des établissements d'enseignement et de recherche français ou étrangers, des laboratoires publics ou privés. 
Page 1 of 14

\title{
Stabilization of a Class of Switched Dynamic Systems: the Riccati Equation Based Approach
}

\author{
M. Bonilla * N.A. Aguillón, M.A. Ortiz Castillo † Jean Jacques.loiseau \\ $\ddagger$ M. Malabre $\S$ V. Azhmyakov $\mathbb{I}$ and S. Salazar $\|$
}

Our paper deals with the stabilization of a class of time-dependent linear autonomous complex systems with a switched structure. The initially given switched dynamic system is assumed to be controlled by a specific state feedback strategy associated with the Linear-QuadraticRegulator (LQR) type control. The proposed control design guarantees stabilization of the closed-loop system for all of the possible location transitions. In the solution procedure of the Algebraic Riccati Equation (ARE) related to the LQR control strategy, only the knowledge of the algebraic structure related to the switched system are needed. We prove that the proposed optimal LQR type state feedback control design stabilizes the closed-loop switched system for every possible active location. The theoretical approach proposed in this paper is finally applied to a model of the Single Wing Quadrotor Aircraft ( $S W Q A$ ), when changing from its Quadrotor Flight Envelope $(Q F E)$ to its Airplane Flight Envelope (AFE).

Keywords: switched dynamic systems, implicit control systems, linear quadratic regulator (LQR), algebraic Riccati equation (ARE), Lyapunov stability.

\section{INTRODUCTION}

General Switched Dynamic Systems (SDSs) belong to a wide family of the Complex Systems studied by many authors (see e.g., (Karcanias and Livada, 2019)). Since SDSs constitute a useful modelling approach for various engineering systems and processes, many useful control strategies for these dynamics have been developed. For example, in (Bonilla et al, 2015a) was shown that a wide class of time-dependent autonomous systems with a switched structure (as defined in (Liberzon, 2003)) can be adequately represented by the formal state space representation of the type

$$
\frac{\mathrm{d}}{\mathrm{d} t} x=A_{q} x+B u
$$

*M. Bonilla, CINVESTAV-IPN, Control Automático, UMI 3175 CINVESTAV-CNRS. A.P. 14-740. México 07000, "mbonilla@cinvestav.mx".

${ }^{\dagger}$ N.A. Aguillón is a Ph.D. student and M.A. Ortiz Castillo was a Master of Science student, both at CINVESTAV-IPN, and sponsored by CONACYT México, "nestor.aguillon@cinvestav.mx", "mortiz@ctrl.cinvestav.mx".

${ }^{\ddagger}$ Jean Jacques.Loiseau, CNRS, LS2N (Laboratoire des Sciences du Numérique de Nantes), UMR 6004, B.P. 92101, 44321 Nantes, Cedex 03, France. "Jean-Jacques.Loiseau@ls2n.fr" .

$\S$ This work was done while M. Malabre was member of LS2N (Laboratoire des Sciences du Numérique de Nantes), UMR 6004, B.P. 92101, 44321 Nantes, Cedex 03, "Michel.Malabre@univ-nantes.fr".

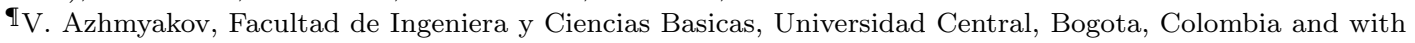
the Institut für Theoretische Informatik, Mathematik und Operations Research, Universität der Bundeswehr München München, Germany "vazhmyakova@ucentral.edu.co" .

"S. Salazar, CINVESTAV-IPN, Sistemas Autónomos de Navegación Aérea Y Submarina, UMI 3175 CINVESTAV-CNRS. A.P. 14-740. México 07000. "sergio.salazar.cruz@gmail.com" . 
Here $B \in \mathbb{R}^{\bar{n} \times m}$ is an injective matrix and $A_{q}$ has the common structure (see e.g., (Narendra $e t$ al, 1994)):

$$
A_{q}=\bar{A}_{0}+\bar{A}_{1} \bar{D}(q)
$$

where $\bar{A}_{0} \in \mathbb{R}^{\bar{n} \times \bar{n}}, \bar{A}_{1} \in \mathbb{R}^{\bar{n} \times \hat{n}}$ is an injective matrix and $\bar{D}(q) \in \mathbb{R}^{\hat{n} \times \bar{n}}$ are surjective matrices. ${ }^{1}$ Let us denote $n=\bar{n}+\hat{n}$.

Due to the usual dynamics of a SDS, it remains in a specific location

$$
q \in \mathscr{Q} \triangleq\left\{q_{1}, \ldots, q_{\eta} \mid q_{i} \in \mathbb{R}^{\mu}, \quad i \in\{1, \ldots, \eta\} \subset \mathbb{Z}^{+}\right\}
$$

for all time instants $t \in \mathscr{I}_{i}$. Here $\mathscr{I}_{i} \triangleq\left[t_{i-1}, t_{i}\right), t_{i} \in \mathbb{R}^{+}, t_{0}=0, t_{i-1}<t_{i}$, for all $i \in \mathbb{Z}^{+}, \lim _{i \rightarrow \infty} T_{i}$ $=\infty$, and $\mathfrak{s}:\left\{\mathscr{I}_{i} \subset \mathbb{R}^{+} \cup\{0\}, i \in \mathbb{Z}^{+}\right\} \rightarrow \mathscr{Q}, \mathfrak{s}\left(\mathscr{I}_{i}\right)=q_{j}, j \in\{1, \ldots, \eta\}$.

We now assume that the locations set has a specific structure described by the following basic Hypothesis.

Hypothesis 1.1 (Bonilla et al, 2015b) Given $\bar{q}_{0}, \bar{q}_{1}, \ldots, \bar{q}_{\ell} \in \mathbb{R}^{\mu}$, the locations $q_{j} \in \mathscr{Q}, j \in$ $\{1, \ldots, \eta\}$, belong to the set described as follows

$$
q_{j} \in \overline{\mathscr{Q}}_{\bar{q}_{0}} \triangleq\left\{q_{j} \in \mathscr{Q} \mid q_{j}=\bar{q}_{0}+\left[\begin{array}{lll}
\bar{q}_{1} & \cdots & \bar{q}_{\ell}
\end{array}\right] \bar{\gamma}_{j}, \bar{\gamma}_{j} \in \mathbb{R}^{\ell}, j \in\{1, \ldots, \eta\}\right\},
$$

and additionally for each $\mathscr{I}_{i} \in \mathfrak{s}^{-1}\left(q_{j}\right), \bar{\gamma}_{j}=\left[\gamma_{j, 1} \cdots \gamma_{j, \ell}\right]^{T}$ take constant values in $\mathbb{R}^{\ell} ; i \in \mathbb{N}, j$ $\in\{1, \ldots, \eta\}$.

Hypothesis 1.2 (Bonilla et al, 2015b) There exist $\bar{\Delta}_{0}, \bar{\Delta}_{\bar{\gamma}_{j}} \in \mathbb{R}^{\hat{n} \times \bar{n}}, j \in\{1, \ldots, \eta\}$, such that

$$
\bar{D}(q)=\bar{\Delta}_{0}-\sum_{i \in \mathbb{N}} \mathbf{1}_{\mathscr{S}_{i}}(t) \bar{\Delta}_{\overline{\mathfrak{s}}\left(\mathscr{S}_{i}\right)}
$$

where $\mathbf{1}_{\mathscr{S}_{i}}(t)$ is the characteristic function of the time interval $\mathscr{I}_{i}$, and $\overline{\mathfrak{s}}:\left\{\mathscr{I}_{i} \subset \mathbb{R}^{+} \cup\{0\}, i \in \mathbb{Z}^{+}\right\}$ $\rightarrow\left\{\bar{\gamma}_{1}, \ldots, \bar{\gamma}_{\ell}\right\}, \overline{\mathfrak{s}}\left(\mathscr{I}_{i}\right)=\bar{\gamma}_{j}, j \in\{1, \ldots, \eta\} . \mathfrak{s}\left(\mathscr{I}_{i}\right)$ and $\overline{\mathfrak{s}}\left(\mathscr{I}_{i}\right)$ follow the same index assignation rule, $i \mapsto j$.

We also assume

Hypothesis 1.3 The pair $\left(\bar{A}_{0}+\bar{A}_{1} \bar{\Delta}_{0}, B\right)$ is controllable.

In (Bonilla et al, 2015b) authors additionally propose a specific variable structure decoupling control strategy based on the ideal proportional and derivative (PD) feedback control strategy. As next a proper practical approximation of the above ideal PD feedback is developed. Such

\footnotetext{
${ }^{1}$ Recall that the celebrated rank-nullity theorem defines the concept of a surjective and an injective matrix. In the case of a $r_{1} \times r_{2}$ matrix with a rank $r_{3}$, this fundamental theorem establishes that

$$
\operatorname{dim} \operatorname{ker} \mathscr{A}=r_{2}-r_{3},
$$

where $\mathscr{A}$ denotes the linear map associated with the given matrix. Injectivity of the matrix is defined as

$$
\operatorname{dim} \operatorname{ker} \mathscr{A}=0 \Rightarrow r_{2}=r_{3}
$$

and the surjectivity is equivalent to $r_{3}=r_{1}$.
} 
feedback control strategies reject the initially given "variable structure" of the resulting system and make it possible to establish the required stability property for control strategies under consideration.

In this paper, we consider the stabilizing problem for a class of time-dependent switched dynamic systems equipped with a relative simple static state feedback. Our paper is organized as follows: in Section 2 we give a formal description of the $L Q$ based stabilization of switched systems. The initial system description is proposed in (Ortiz et al, 2020). We next follow the more formal approach discussed in (Bonilla et al, 2015a). In Section 3 we apply the celebrated Riccati stabilizing state feedback (see also Section 2) for a control design that stabilizes a Single Wing Quadrotor Aircraft in the case when changing from its Quadrotor Flight Envelope to its Airplane Flight Envelope. Section 4 summarizes our paper.

\section{RICCATI EQUATION BASED APPROACH TO THE STABILIZATION PROBLEM}

Let us formulate the following problem.

Problem 2.1 Find a constant state feedback which stabilizes system (1.1) and (1.2),

$$
\frac{\mathrm{d}}{\mathrm{d} t} x=\left(\bar{A}_{0}+\bar{A}_{1} \bar{D}(q)\right) x+B u .
$$

We next assume that the locations (1.3) are unknown

$$
q \in \mathscr{Q} \triangleq\left\{q_{1}, \ldots, q_{\eta} \mid q_{i} \in \mathbb{R}^{\mu}, \quad i \in\{1, \ldots, \eta\} \subset \mathbb{Z}^{+}\right\} .
$$

We also assume the full knowledge of the essential parameters of constant system structure, (1.2) and (1.5), determined by the following triplet $\left(\bar{A}_{0}, \bar{A}_{1}, \bar{\Delta}_{0}\right)$.

Solution of this problem involves the proposed state feedback with the LQR structure

$$
F_{* 0}=R^{-1} B^{T} P_{0}, \quad u=-F_{* 0} x,
$$

where $R$ is a selected positive definite matrix and $P_{0}$ is a solution of the Algebraic Riccati Equation (ARE) (recall Assumption 1.3)

$$
\left(\bar{A}_{0}+\bar{A}_{1} \bar{\Delta}_{0}\right)^{T} P_{0}+P_{0}\left(\bar{A}_{0}+\bar{A}_{1} \bar{\Delta}_{0}\right)-P_{0} B R^{-1} B^{T} P_{0}+\bar{Q}_{0}=0,
$$

and $\bar{Q}_{0}$ is a selected positive semidefinite matrix. The above ARE is determined by the essential parameters of system (1.1) and (1.2), namely $\left(\bar{A}_{0}, \bar{A}_{1}, \bar{\Delta}_{0}\right)$ (recall that (1.2) and (1.5)).

Applying the state feedback (2.1) to the switched system representation (1.1), we next obtain the closed loop state space form

$$
\frac{\mathrm{d}}{\mathrm{d} t} x=\left(A q_{i}-B R^{-1} B^{T} P_{0}\right) x
$$

Taking into consideration the previously derived formulae (1.2) and (1.5) in (2.3), we also get

$$
\frac{\mathrm{d}}{\mathrm{d} t} x=\left(\bar{A}_{0}+\bar{A}_{1}\left(\bar{\Delta}_{0}-\sum_{i \in \mathbb{N}} \mathbf{1}_{\mathscr{S}_{i}}(t) \bar{\Delta}_{\overline{\mathfrak{s}}\left(\mathscr{S}_{i}\right)}\right)-B R^{-1} B^{T} P_{0}\right) x,
$$


4 of 14

In the same way as in Narendra et al (1994) let us define the following Lyapunov function

$$
V(x)=x(t)^{T} P_{0} x(t) .
$$

The usual Lie derivative of (2.5) (the derivative along the trajectories of system (2.4)) can be calculated as follows

$$
\begin{aligned}
\mathrm{d} V(t) / \mathrm{d} t= & \mathrm{d} x^{T}(t) / \mathrm{d} t P_{0} x+x^{T}(t) P_{0} \mathrm{~d} x / \mathrm{d} t \\
= & x^{T}\left(\left(\bar{A}_{0}+\bar{A}_{1} \bar{\Delta}_{0}\right)^{T} P_{0}-\sum_{i \in \mathbb{N}} \mathbf{1}_{\mathscr{I}_{i}}(t)\left(\bar{A}_{1} \bar{\Delta}_{\overline{\mathfrak{s}}\left(\mathscr{I}_{i}\right)}\right)^{T} P_{0}-\left(B R^{-1} B^{T} P_{0}\right)^{T} P_{0}\right) x \\
& +x^{T}\left(P_{0}\left(\bar{A}_{0}+\bar{A}_{1} \bar{\Delta}_{0}\right)-\sum_{i \in \mathbb{N}} \mathbf{1}_{\mathscr{S}_{i}}(t) P_{0}\left(\bar{A}_{1} \bar{\Delta}_{\overline{\mathfrak{s}}\left(\mathscr{I}_{i}\right)}\right)-P_{0}\left(B R^{-1} B^{T} P_{0}\right)\right) x \\
\mathrm{~d} V(t) / \mathrm{d} t= & x^{T}\left(\left(\bar{A}_{0}+\bar{A}_{1} \bar{\Delta}_{0}\right)^{T} P_{0}+P_{0}\left(\bar{A}_{0}+\bar{A}_{1} \bar{\Delta}_{0}\right)-P_{0} B R^{-1} B^{T} P_{0}\right. \\
& \left.-\sum_{i \in \mathbb{N}} \mathbf{1}_{\mathscr{I}_{i}}(t)\left(\left(\bar{A}_{1} \bar{\Delta}_{\overline{\mathfrak{s}}\left(\mathscr{I}_{i}\right)}\right)^{T} P_{0}+P_{0}\left(\bar{A}_{1} \bar{\Delta}_{\overline{\mathfrak{s}}\left(\mathscr{S}_{i}\right)}\right)\right)-P_{0} B R^{-1} B^{T} P_{0}\right) x .
\end{aligned}
$$

From (2.2) and (2.1) we next deduce

$$
\begin{aligned}
\mathrm{d} V(t) / \mathrm{d} t & =-x^{T}\left(\bar{Q}_{0}+\sum_{i \in \mathbb{N}} 1_{\mathscr{S}_{i}}(t)\left(\left(\bar{A}_{1} \bar{\Delta}_{\overline{\mathfrak{s}}\left(\mathscr{S}_{i}\right)}\right)^{T} P_{0}+P_{0}\left(\bar{A}_{1} \bar{\Delta}_{\overline{\mathfrak{s}}\left(\mathscr{S}_{i}\right)}\right)\right)+F_{* 0}^{T} R F_{* 0}\right) x, \\
\mathrm{~d} V(t) / \mathrm{d} t & =-x^{T}\left(Q_{0}+\sum_{i \in \mathbb{N}} 1_{\mathscr{S}_{i}}(t)\left(\left(\bar{A}_{1} \bar{\Delta}_{\overline{\mathfrak{s}}\left(\mathscr{S}_{i}\right)}\right)^{T} P_{0}+P_{0}\left(\bar{A}_{1} \bar{\Delta}_{\overline{\mathfrak{s}}\left(\mathscr{I}_{i}\right)}\right)\right)\right) x \\
\mathrm{~d} V(t) / \mathrm{d} t & =-x^{T}\left(Q_{0}+\sum_{i \in \mathbb{N}} 1_{\mathscr{S}_{i}}(t) \bar{Q}_{\overline{\mathfrak{s}}\left(\mathscr{I}_{i}\right)}\right) x
\end{aligned}
$$

where

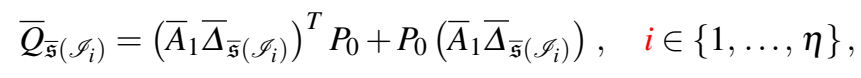

$Q_{0}=\left[\left(\sqrt{\bar{Q}_{0}}\right)^{T} \quad F_{* 0}^{T}\right]\left[\begin{array}{cc}\mathrm{I} & 0 \\ 0 & R\end{array}\right]\left[\begin{array}{c}\sqrt{\bar{Q}_{0}} \\ F_{* 0}\end{array}\right]$, and $\bar{Q}_{0}=\left(\sqrt{\bar{Q}_{0}}\right)^{T}\left(\sqrt{\bar{Q}_{0}}\right)$.

The analytic relations obtained above constitute in fact a formal proof of our main stability result

Theorem 2.2 Assume that all the technical assumptions of this section are fulfilled. Then the system (2.3) is stable in the sense of Lyapunov if one of the two following conditions is satisfied

$$
\lambda_{\min }\left\{Q_{0}+\left(\bar{A}_{0}+\bar{A}_{1} \bar{\Delta}_{0}-A_{q_{j}}\right)^{T} P_{0}+P_{0}\left(\bar{A}_{0}+\bar{A}_{1} \bar{\Delta}_{0}-A_{q_{j}}\right)\right\}>0, \quad \forall j \in\{1, \ldots, \eta\},
$$

or if the pair $\left(\sqrt{\bar{Q}_{0}},\left(\bar{A}_{0}+\bar{A}_{1} \bar{\Delta}_{0}\right)\right)$ is observable and moreover, 


$$
\lambda_{\min }\left\{Q_{0}+\left(\bar{A}_{0}+\bar{A}_{1} \bar{\Delta}_{0}-A_{q_{j}}\right)^{T} P_{0}+P_{0}\left(\bar{A}_{0}+\bar{A}_{1} \bar{\Delta}_{0}-A_{q_{j}}\right)\right\} \geqslant 0, \quad \forall j \in\{1, \ldots, \eta\}
$$

Proof. Let us first note that (1.5), (1.2) and (2.7), imply:

$$
\bar{Q}_{\bar{\gamma}_{j}}=\left(\bar{A}_{1} \bar{\Delta}_{\bar{\gamma}_{j}}\right)^{T} P_{0}+P_{0}\left(\bar{A}_{1} \bar{\Delta}_{\bar{\gamma}_{j}}\right)=\left(\bar{A}_{0}+\bar{A}_{1} \bar{\Delta}_{0}-A_{q_{j}}\right)^{T} P_{0}+P_{0}\left(\bar{A}_{0}+\bar{A}_{1} \bar{\Delta}_{0}-A_{q_{j}}\right), \quad j \in\{1, \ldots, \eta\} .
$$

Application of Theorem 5.10 from Chapter 6, Section 5 of Stewart (1973), and Corollary 2.6-2 of Kailath (1980)) concludes the proof.

REMARK 2.1 : The obtained result provides a stability criterion for the switched systems in the absence of the exact (a priori given) information about a concrete switching mechanism.

Starting from a model given in the form (1.1), the feedback design procedure can next be summarized as follows.

1. Identify the essential parameters of the constant system structure, (1.2) and (1.5), $\left(\bar{A}_{0}\right.$, $\left.\bar{A}_{1}, \bar{\Delta}_{0}\right)$.

2. Choose matrices $R$ and $\bar{Q}_{0}$ and solve the Riccatti equation (2.2).

3. If one of the two conditions of Theorem 2.2, (2.8) or (2.9), are satisfied go to next item, otherwise return to the previous item.

4. Define the specific feedback control law by $(2.1)$.

\section{A PRACTICAL EXAMPLE}

Let us consider the Single Wing Quadrotor Aircraft (SWQA) as shown in Fig. 1. The mechanical motion of the $S W Q A$ is studied in a fixed orthogonal axis set (earth axes) (OXYZ), where $O \mathbf{Z}$ is a vertical axis, along the gravity vector $\left[\begin{array}{ccc}0 & 0 & g\end{array}\right]^{T}$.

Let $\Phi, \Theta$ and $\Psi$ be the conventional Euler angles, roll, pitch and yaw, measured with respect to the axis $O_{B} \mathbf{X}_{B}, O_{B} \mathbf{Y}_{B}$ and $O_{B} \mathbf{Z}_{B}$. Here $\left(O_{B} \mathbf{X}_{B} \mathbf{Y}_{B} \mathbf{Z}_{B}\right)$ is the body axis system with its origin $O_{B}$ fixed at the centre of gravity of the $S W Q A$ (Cook, 2013). The total mass of the quadrotor of the $S W Q A$ is equal to $m=1.6[\mathrm{~kg}]$ and the moments of inertia with respect to the axis $O_{B} \mathbf{X}_{B}, O_{B} \mathbf{Y}_{B}$ and axis $O_{B} \mathbf{Z}_{B}$ are $\mathrm{I}_{x x}=0.058\left[\mathrm{~kg} \mathrm{~m}^{2}\right], \mathrm{I}_{y y}=0.048\left[\mathrm{~kg} \mathrm{~m}^{2}\right]$ and $\mathrm{I}_{z z}=0.052\left[\mathrm{~kg} \mathrm{~m}^{2}\right]$, respectively. We consider here some concrete values: for the gravity $g=9.81\left[\mathrm{~m} \mathrm{~s}^{-2}\right]$ and the air density parameter $\rho=1.2\left[\mathrm{~kg} / \mathrm{m}^{3}\right]$.

Moreover, we assume that the single wing has a S5010 low speed airfoil for flying wings (Selig et al, 1996), with the aspect ratio: $A R=6$, span $b=1.35[\mathrm{~m}]$ and mean aerodynamic chord $\overline{\bar{c}}$ $=0.165$. Let $[\mathrm{m}]$ be a distance of the c.g. along $\overline{\bar{c}}: h=0.1$ and location of the aerodynamic center $h_{0} \approx 0.25$. 
6 of 14

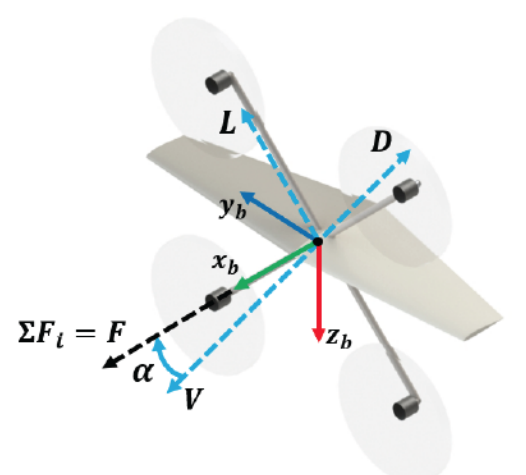

(a)

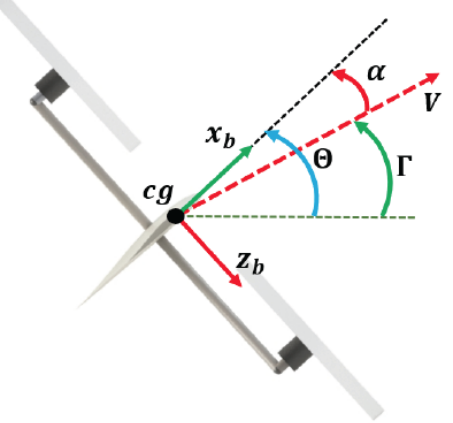

(b)

FIG. 1. Single wing quadrotor aircraft. (a) Perspective view. (b) Tangential variables, $\Gamma$ : flight path angle, $\Theta$ : pitch angle, $\boldsymbol{\alpha}$ : angle of attack, $V$ : longitudinal flight speed.

\subsection{Description of the Longitudinal Directional Behavior}

We next assume that the lateral directional dynamics ( $\mathbf{X}-\mathbf{Y}$ plane) has been already asymptotically stabilized. Thus, we only need to consider the longitudinal directional movement $(\mathbf{X}-\mathbf{Z}$ plane). The SWQA is represented by the following differential equations:

$$
\begin{aligned}
{\left[\begin{array}{l}
\mathrm{d}^{2} X / \mathrm{d} t^{2} \\
\mathrm{~d}^{2} Z / \mathrm{d} t^{2}
\end{array}\right] } & =\left[\begin{array}{l}
0 \\
g
\end{array}\right]-(1 / m)\left[\begin{array}{cc}
\cos \Gamma & \sin \Gamma \\
-\sin \Gamma & \cos \Gamma
\end{array}\right]\left[\begin{array}{l}
D(V, \boldsymbol{\alpha}) \\
L(V, \boldsymbol{\alpha})
\end{array}\right]+(F / m)\left[\begin{array}{c}
\cos \Theta \\
-\sin \Theta
\end{array}\right], \\
\mathrm{d}^{2} \Theta / \mathrm{d} t^{2} & =\left(1 / \mathrm{I}_{y y}\right)\left(T_{q}+M(V, \boldsymbol{\alpha})+\overline{\bar{c}}\left(h-h_{0}\right) L(V, \boldsymbol{\alpha})\right),
\end{aligned}
$$

where the lift, drag and pitching moment equations of the wing are given by the following relations (see (Cook, 2013) for details):

$$
L(V, \boldsymbol{\alpha})=\frac{1}{2} \rho \frac{b^{2}}{A R} V^{2} C_{L}(\boldsymbol{\alpha}), \quad D(V, \boldsymbol{\alpha})=\frac{1}{2} \rho \frac{b^{2}}{A R} V^{2} C_{D}(\boldsymbol{\alpha}), \quad M(V, \boldsymbol{\alpha})=\overline{\bar{c}} \frac{1}{2} \rho \frac{b^{2}}{A R} V^{2} C_{M}(\boldsymbol{\alpha})
$$

Here $V$ is the flying speed calculated by

$$
V=\sqrt{(\mathrm{d} \mathbf{X} / \mathrm{d} t)^{2}+(\mathrm{d} \mathbf{Z} / \mathrm{d} t)^{2}} .
$$

Assuming now the absence of wind and denote by $\boldsymbol{\alpha}$ the angle of attack, defined as the angle between the chord line of the airfoil and the velocity vector. Clearly, it is related to the pitch angle $\Theta$

$$
\Theta=\boldsymbol{\alpha}+\Gamma, \quad \Gamma=\arctan \left(\frac{-\mathrm{d} \mathbf{Z} / \mathrm{d} t}{\mathrm{~d} \mathbf{X} / \mathrm{d} t}\right),
$$

where $\Gamma$ is called the flight path angle and defined as the angle between the velocity vector and the horizontal plane (Cook, 2013, p. 18) (c.f Fig. 1). The dimensionless aerodynamic coefficients $C_{L}, C_{D}, C_{M}$ in (3.2) has the classic expressions:

$$
C_{L}(\boldsymbol{\alpha})=C_{L_{0}}+C_{L_{1}} \boldsymbol{\alpha}, C_{D}(\boldsymbol{\alpha})=C_{D_{0}}+C_{D_{1}} \boldsymbol{\alpha}+C_{D_{2}} \boldsymbol{\alpha}^{2}, C_{M}(\boldsymbol{\alpha})=C_{M_{0}}+C_{M_{1}} \boldsymbol{\alpha},
$$

where $\left(C_{L_{0}}, C_{L_{1}}\right)=(0.1875,0660),\left(C_{D_{0}}, C_{D_{1}}, C_{D_{2}}\right)=(0.0212,0.0014,0.0004)$ and $\left(C_{M_{0}}, C_{M_{1}},\right)=$ $(-0.0134,0.0092)$. 
The input forces and moments in the body frame are obtained from the angular velocities of the existing four rotors: $\omega_{i}, i=1, \ldots 4$ according to

$$
\left[\begin{array}{c}
F \\
T_{p} \\
T_{q} \\
T_{r}
\end{array}\right]=\left[\begin{array}{cccc}
k_{f} & k_{f} & k_{f} & k_{f} \\
k_{\tau} & k_{\tau} & -k_{\tau} & -k_{\tau} \\
-k_{q} & k_{q} & k_{q} & -k_{q} \\
-k_{r} & k_{r} & -k_{r} & k_{r}
\end{array}\right]\left[\begin{array}{c}
\omega_{1}^{2} \\
\omega_{2}^{2} \\
\omega_{3}^{2} \\
\omega_{4}^{2}
\end{array}\right]
$$

where $F$ is the input thrust, $T_{p}, T_{q}$ and $T_{r}$ are the input torques of the rotors along the body axes and $k_{f}, k_{\tau}$ are the force and torque coefficients of the rotors, respectively. Additionally $k_{q}$ and $k_{r}$ are torque coefficients derived from the rotor configuration shown in Fig. 1 (Powers et $a l, 2014)$.

\subsection{Problem Statement}

Consider the $S W Q A$ equiped with the four rotors vertically oriented in a hover flight $\Theta=90^{\circ}$ (as shown in Fig. 1(b)). In fact we are dealing with the Quadrotor Flight Envelope $(Q F E)$ and want to change the quadrotor mode into an airplane mode. That means the $S W Q A$ has to fly as an airplane in an horizontal flying path $\Gamma=0^{\circ}$ with a positive angle of attack $\boldsymbol{\alpha}$. This angle needs to be not larger than $10^{\circ}$ and the flying speed $V$ is predefined to be not larger than 5 $[\mathrm{m} / \mathrm{s}]$. This described flying regime will next be called the Airplane Flight Envelope (AFE). The required change between these two flight modes guarantees that the $S W Q A$ will continue the flight and not fall down.

To solve the problem described above we have to track a take-off path in terms of its nominal tangential velocity. Given a desired flying speed, $\bar{V}$ and a required flight path angle $\bar{\Gamma}$, the $S W Q A$ has to track a prescribed trajectory $(\overline{\mathbf{X}}, \overline{\mathbf{Z}})$ such that

$$
\mathrm{d} \overline{\mathbf{X}} / \mathrm{d} t=\bar{V} \cos (\bar{\Gamma}), \quad \mathrm{d} \overline{\mathbf{Z}} / \mathrm{d} t=-\bar{V} \sin (\bar{\Gamma}) .
$$

In the $Q F E$ the thrust $F$ of the rotors is the main lift force and in the $A F E$ are the wing aerodynamic forces. By $L$ and $D$ we denote the main lift forces. During the transition phase between two flight envelopes mentioned above the importance between two different types of forces changes gradually.

The nominal values of $F$ and $L$ are obtained from (3.1), (3.3), (3.4) and (3.7) as

$$
\left[\begin{array}{c}
\bar{F} \\
\bar{L}
\end{array}\right]=\left[\begin{array}{c}
D(\bar{V}, \overline{\boldsymbol{\alpha}}) / \cos (\overline{\boldsymbol{\alpha}}) \\
-D(\bar{V}, \overline{\boldsymbol{\alpha}}) \tan (\overline{\boldsymbol{\alpha}})
\end{array}\right]+(m g)\left[\begin{array}{c}
\sin (\bar{\Gamma}) / \cos (\overline{\boldsymbol{\alpha}}) \\
\cos (\bar{\Gamma})-\sin (\bar{\Gamma}) \tan (\overline{\boldsymbol{\alpha}})
\end{array}\right]-m\left[\begin{array}{c}
-(\mathrm{d} \bar{V} / \mathrm{d} t) / \cos (\overline{\boldsymbol{\alpha}}) \\
(\mathrm{d} \bar{V} / \mathrm{d} t) \tan (\overline{\boldsymbol{\alpha}})-\bar{V}(\mathrm{~d} \bar{\Gamma} / \mathrm{d} t)
\end{array}\right],
$$

where: $\bar{V}=\sqrt{(\mathrm{d} \overline{\mathbf{X}} / \mathrm{d} t)^{2}+(\mathrm{d} \overline{\mathbf{Z}} / \mathrm{d} t)^{2}}$ and $(\cos (\overline{\boldsymbol{\alpha}}) \approx 1$ and $\sin (\overline{\boldsymbol{\alpha}}) \approx \overline{\boldsymbol{\alpha}})$ :

$$
\overline{\boldsymbol{\alpha}} \approx \frac{(m g) \cos (\bar{\Gamma})+m \bar{V} \mathrm{~d} \bar{\Gamma} / \mathrm{d} t-(1 / 2) \rho\left(b^{2} / A R\right) \bar{V}^{2} C_{L_{0}}}{(1 / 2) \rho\left(b^{2} / A R\right) \bar{V}^{2}\left(C_{L_{1}}+C_{D_{0}}\right)+(m g) \sin (\bar{\Gamma})+m \mathrm{~d} \bar{V} / \mathrm{d} t} .
$$

Note that the nominal value of the pitching moment $T_{q}$ is given by

$$
\bar{T}_{q}=\mathrm{I}_{y y} \mathrm{~d}^{2} \bar{\Gamma} / \mathrm{d} t^{2}-M(\bar{V}, \overline{\boldsymbol{\alpha}})-\overline{\bar{c}}\left(h-h_{0}\right) \bar{L}(\bar{V}, \overline{\boldsymbol{\alpha}}) .
$$


8 of 14

Using these predetermined nominal values we are able to define the longitudinal incremental variables given as follows

$$
\mathbf{x}=\overline{\mathbf{X}}-\mathbf{X}, \quad \mathbf{z}=\overline{\mathbf{Z}}-\mathbf{Z}, \quad \theta=\bar{\Theta}-\Theta, \quad f=\bar{F}-F, \quad \tau_{q}=\bar{T}_{q}-T_{q} .
$$

\subsection{Problem Solution Method}

In order to use the constructive treatment of the problem established in the previous section let us first define the following smooth take-off path

$$
\bar{V}=\bar{V}_{0}+\frac{\bar{V}_{N}-\bar{V}_{0}}{2}\left(1-\cos \left(\pi \frac{t}{t_{N}}\right)\right)[\mathrm{m} / \mathrm{s}], \quad \bar{\Gamma}=\frac{\pi}{2}-\frac{\pi}{4}\left(1-\cos \left(\pi \frac{t}{t_{N}}\right)\right)[\mathrm{rad}],
$$

where $t_{N}$ is the transition time, $\bar{V}_{0}$ is the initial speed and $\bar{V}_{N}$ denotes the desired cruise speed (see Fig. 2).

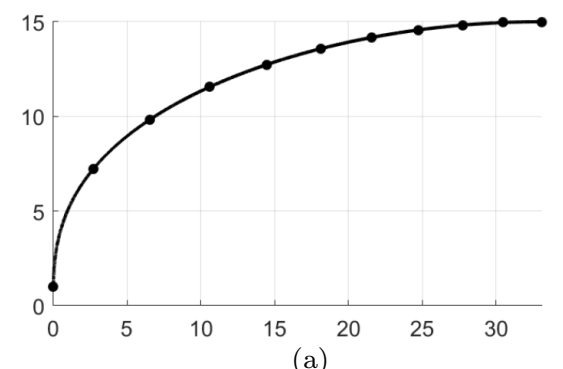

(a)
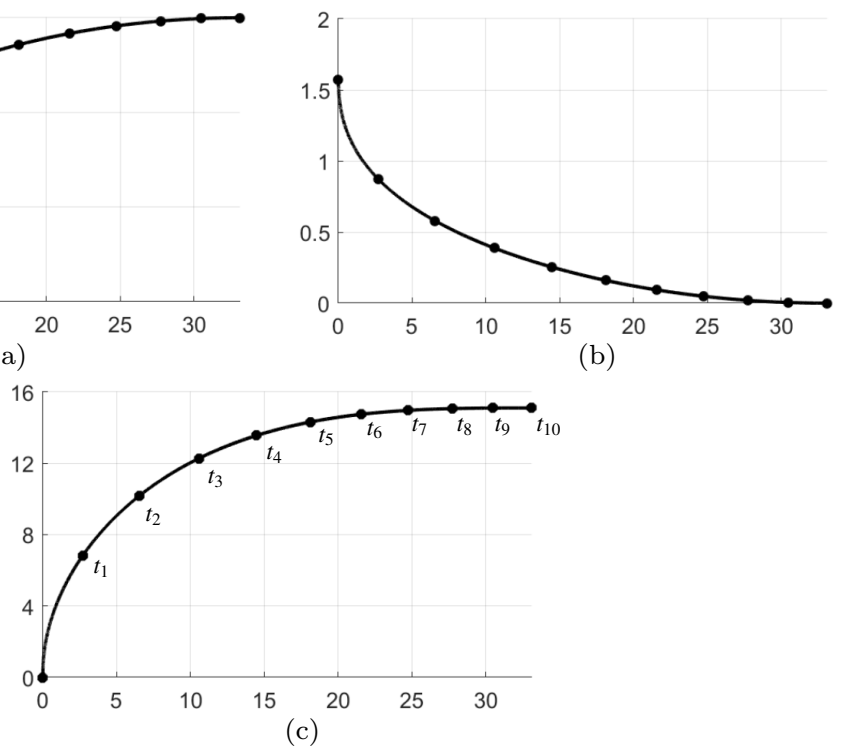

FIG. 2. Take-off path (3.12) and (3.7); $\bar{V}_{0}=1[\mathrm{~m} / \mathrm{s}], \bar{V}_{N}=15[\mathrm{~m} / \mathrm{s}], t_{N}=5[\mathrm{~m} / \mathrm{s}]$. (a) $\bar{V}[\mathrm{~m} / \mathrm{s}]$ v.s. $\overline{\mathbf{X}}[\mathrm{m}]$. (b) $\bar{\Gamma}[\mathrm{rad}]$ v.s. $\overline{\mathbf{X}}[\mathrm{m}] .(\mathrm{c})-\overline{\mathbf{Z}}[\mathrm{m}]$ v.s. $\overline{\mathbf{X}}[\mathrm{m}]$.

We next consider a necessary time partition: $\mathscr{P}_{10}=\left\{\mathscr{I}_{1}, \ldots, \mathscr{I}_{N}\right\}, \mathscr{I}_{k}=\left[t_{k-1}, t_{k}\right), t_{0}=0$, $t_{k-1}<t_{k}, k \in\{1, \ldots, N\}$, and introduce the specific change of variable:

$$
\left[\begin{array}{l}
\hat{\mathbf{x}} \\
\hat{\mathbf{z}}
\end{array}\right]=P\left[\begin{array}{l}
\mathbf{x} \\
\mathbf{z}
\end{array}\right], \quad P=\left[\begin{array}{cc}
\cos (\widehat{\boldsymbol{\Theta}}(t)) & -\sin (\widehat{\boldsymbol{\Theta}}(t)) \\
\sin (\widehat{\boldsymbol{\Theta}}(t)) & \cos (\widehat{\boldsymbol{\Theta}}(t))
\end{array}\right] ; \quad \widehat{\boldsymbol{\Theta}}(t)=\sum_{k=1}^{N} \mathbf{1}_{\mathscr{F}_{k}}(t) \overline{\boldsymbol{\Theta}}\left(t_{k}\right) .
$$

From (3.1), (3.11) and (3.13), we can deduce the useful state space description for the input and state vectors

$$
\frac{\mathrm{d}}{\mathrm{d} t} x=A_{q} x+B u+S \mathrm{q}(x),
$$




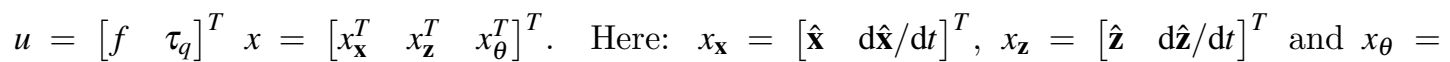
$\left[\begin{array}{ll}\theta & \mathrm{d} \theta / \mathrm{d} t\end{array}\right]^{T} ; \mathrm{q}(x)=\left[\begin{array}{lll}\mathrm{q}_{\mathrm{x} k} & \mathrm{q}_{\mathrm{zk}} & \mathrm{q}_{\theta k}\end{array}\right]^{T}$ is the uncertainty vector (nonlinear perturbation signal) (Bonilla et al, 2020). This vector describes the terms that are neglected when a linearization around the equilibrium points $(x, u)=(0,0)$ is implemented. Moreover, we need to introduce matrices $A_{q}, B$ and $S$

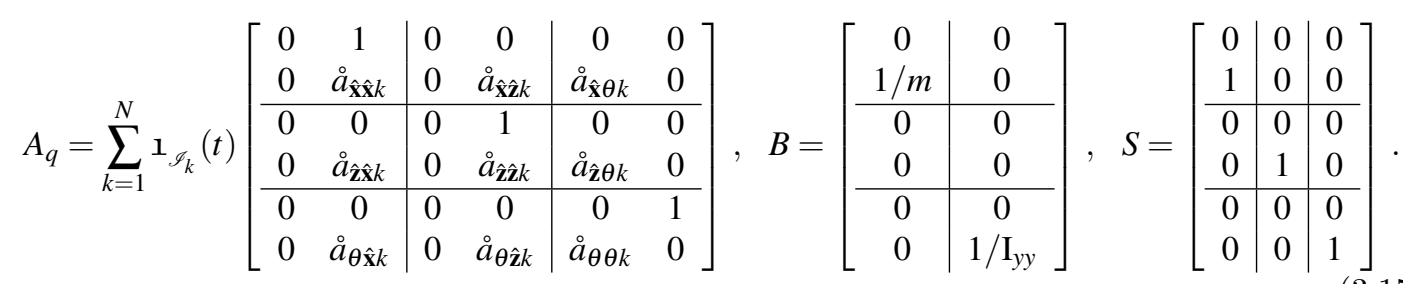

The $\stackrel{\circ}{a}_{i j k}, i, j \in\{\hat{\mathbf{x}}, \hat{\mathbf{z}}, \theta\}$ are constant coefficients determined over the intervals $\mathscr{I}_{k}$ for $k \in$ $\{1, \ldots, N\}$. These coefficients are results of the linearizing procedure (3.1), (3.11) and (3.13) realised over the take-off path (3.12) and (3.7). This linearization is considered at the time instants $\left\{t_{1}, \ldots, t_{N}\right\}$ as shown in Fig. 2. Let us note that Appendix 5 contains the the concrete necessary algebraic expressions we applied here.

\subsection{Riccati Stabilization}

In order to tackle the Riccati Stabilization of (3.14) and (3.15) we first definine the locations set $(c f .(1.3))$

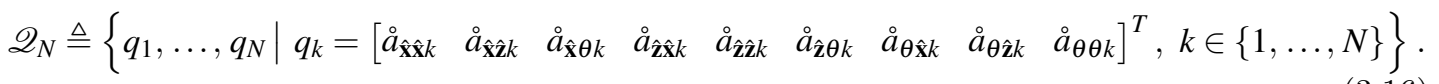

Thus, matrix $A_{q}$ takes the following form (cf. (1.2a)):

$$
\begin{aligned}
& A_{q}=\bar{A}_{0}+\bar{A}_{1} \bar{D}(q), \quad \bar{D}(q)=\sum_{k=1}^{N} \mathbf{1}_{\mathscr{l}_{k}}(t) \bar{D}\left(q_{k}\right), \quad A_{q_{k}}=\bar{A}_{0}+\bar{A}_{1} \bar{D}\left(q_{k}\right), \\
& \bar{A}_{0}=\left[\begin{array}{cc|cc|cc}
0 & 1 & 0 & 0 & 0 & 0 \\
0 & 0 & 0 & 0 & 0 & 0 \\
\hline 0 & 0 & 0 & 1 & 0 & 0 \\
0 & 0 & 0 & 0 & 0 & 0 \\
\hline 0 & 0 & 0 & 0 & 0 & 1 \\
0 & 0 & 0 & 0 & 0 & 0
\end{array}\right], \bar{A}_{1}=\left[\begin{array}{ccc}
0 & 0 & 0 \\
1 & 0 & 0 \\
\hline 0 & 0 & 0 \\
0 & 1 & 0 \\
\hline 0 & 0 & 0 \\
0 & 0 & 1
\end{array}\right],
\end{aligned}
$$

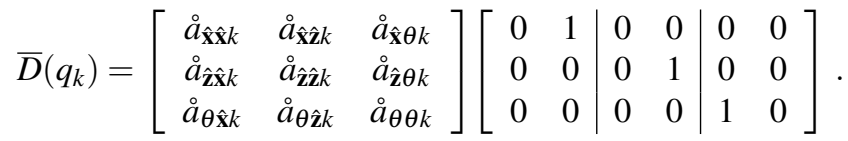

We next define the time partition $\left(N=10\right.$ and $\left.t_{N}=5[\mathrm{~s}]\right)$ as

$$
\mathscr{P}_{10} \triangleq\left\{\mathscr{I}_{k}=\left[t_{k-1}, t_{k}\right) \mid t_{0}=0, t_{k}=5(k / 10)^{(475 / 1918)}, k \in\{1, \ldots, 10\}\right\} .
$$

Using the introduced time partition we get the locations shown in Table 1 (cf. (3.16). We also refer to Appendix 5 for the necessary technical details. 
10 of 14

Table 1. Numerical values of the components $\stackrel{\circ}{a}_{i j k}, i, j \in\{\hat{\mathbf{x}}, \hat{\mathbf{z}}, \theta\}$, of the locations $q_{k} \in \mathscr{Q}_{10}, k \in\{1, \ldots, 10\}$ (cf. (3.16); see also Appendix 5). The column $(\overline{\mathbf{X}},-\overline{\mathbf{Z}})$ was rounded to hundredth and the columns of the $\stackrel{\circ}{a}_{i j k}$ were rounded to thousandth.

\begin{tabular}{|c|c|c|c|c|c|c|c|c|c|c|}
\hline$(\overline{\mathbf{X}},-\overline{\mathbf{Z}})[\mathrm{m}]$ & $k$ & $-\stackrel{\circ}{a}_{\hat{\mathbf{x}} \hat{\mathbf{x}} k}$ & $\stackrel{\circ}{\mathbf{x}} \hat{\mathbf{z}} \hat{z}$ & $-\stackrel{\circ}{a}_{\hat{\mathbf{x}} \theta k}$ & $-\stackrel{\circ}{a} \hat{\mathbf{z}} \mathbf{x} k$ & $-\stackrel{\circ}{a} \hat{\mathbf{z}} \mathbf{k}$ & $-\stackrel{\circ}{a}_{\hat{\mathbf{z}} \theta k}$ & $-\stackrel{\circ}{a}_{\theta \hat{\mathbf{x}} k}$ & $-\stackrel{\circ}{a}_{\theta \mathbf{z} k}$ & $-\stackrel{\circ}{\theta \theta}_{\theta}$ \\
\hline$(5.79,9.67)$ & 1 & .041 & $\overline{0.279}$ & 0.435 & 0.525 & 4.049 & 248149 & 0.429 & 2.221 & 2.207 \\
\hline$(10.68,12.32)$ & 2 & & 0.274 & 0.846 & 0.592 & 4.969 & & .538 & 15 & 3.333 \\
\hline$(14.80,13.64)$ & 3 & 058 & 0.292 & 1.071 & 0.647 & 5.499 & 76. & 0.592 & 4.09 & 4.085 \\
\hline$(18.36,14.35)$ & 4 & 061 & 0.325 & 1.161 & 0.698 & 5.842 & 83.623 & 0.616 & 4.622 & 4.608 \\
\hline$(21.48,14.74)$ & 5 & 33 & 0.367 & 1.157 & 0.748 & 6.075 & & 0.621 & 4.994 & 4.977 \\
\hline$(24.26,14.94)$ & 6 & 064 & 0.414 & 1.0 & $\overline{0.7}$ & 6.233 & & 0.612 & 5.253 & 5.232 \\
\hline$(26.77,15.04)$ & 7 & J64 & 0.463 & 0.974 & 0.842 & 6.340 & 94.756 & 0.594 & 5.429 & 5.405 \\
\hline$(29.05,15.09)$ & 8 & 064 & 0.515 & 0.829 & 0.887 & 6.409 & 96.079 & 0.571 & 5.540 & 5.512 \\
\hline$(31.14,15.10)$ & c & & & & & 84 & 6644 & .544 & 5.600 & 5.569 \\
\hline$(33.07,15.10)$ & & 0.062 & 0.619 & 0.486 & 0.969 & 6.467 & 96.614 & 0.515 & 5.621 & 5.586 \\
\hline
\end{tabular}

From (3.17) and Table 1 we next obtain the spectra of the matrix $A_{q_{k}}$ :

$$
\sigma\left(A_{q_{k}}\right)=\left\{\begin{array}{cll}
q_{1}: & \{0,0,3.4869,-0.099159,-3.7391+3.7709 l,-3.7391-3.7709 \imath\} \\
q_{2}: & \{0,0,4.4614,-0.099919,-4.6913+4.8079 l,-4.6913-4.8079 \imath\} \\
q_{3}: & \{0,0,5.0611,-0.10296,-5.2577+5.4413 \imath,-5.2577-5.4413 \imath\} \\
q_{4}: & \{0,0,5.4595,-0.10605,-5.6286+5.8614 l,-5.6286-5.8614 \imath\} \\
q_{5}: & \{0,0,5.7299,-0.10869,-5.8795+6.1473 \imath,-5.8795-6.1473 \imath\} \\
q_{6}: & \{0,0,5.9117,-0.11077,-6.0491+6.3408 \imath,-6.0491-6.3408 \imath\} \\
q_{7}: & \{0,0,6.0291,-0.11231,-6.1606+6.4674 \imath,-6.1606-6.4674 \imath\} \\
q_{8}: & \{0,0,6.0979,-0.11332,-6.2287+6.544 \imath,-6.2287-6.544 \imath\} \\
q_{9}: & \{0,0,6.1292,-0.11386,-6.2636+6.582 \imath,-6.2636-6.582 \imath\} \\
q_{10}: & \{0,0,6.1312,-0.11397,-6.2728+6.59 \imath,-6.2728-6.59 \imath\}
\end{array}\right.
$$

We now consider the concrete solution procedure for ARE (2.2). In order to solve this ARE we put

$$
q_{0}=q_{10} \quad \Rightarrow \quad \bar{\Delta}_{0}=\bar{D}\left(q_{10}\right)
$$

and choose

$$
\bar{Q}_{0}=\left[\begin{array}{llllll}
1 & 0 & 0 & 0 & 0 & 0 \\
0 & 1 & 0 & 0 & 0 & 0 \\
0 & 0 & 1 & 0 & 0 & 0 \\
0 & 0 & 0 & 1 & 0 & 0 \\
0 & 0 & 0 & 0 & 2 & 0 \\
0 & 0 & 0 & 0 & 0 & 2
\end{array}\right] \text { and } R=\left[\begin{array}{cc}
1 / 20 & 0 \\
0 & 1
\end{array}\right] .
$$

From (3.17), (3.20) and taking into consideration Table 1 and (3.21), we can deduce that the 
Table 2. Spectra $\sigma\left(X_{k}\right), X_{k}=\left(\bar{A}_{0}+\bar{A}_{1} \bar{\Delta}_{0}-A_{q_{k}}\right)^{T} P_{0}+P_{0}\left(\bar{A}_{0}+\bar{A}_{1} \bar{\Delta}_{0}-A_{q_{k}}\right)$ and condition $(2.8)$ for $k \in\{1, \ldots, 10\}$; values rounded to ten thousandth.

\begin{tabular}{|c||c|c|}
\hline$k$ & $\sigma\left(X_{k}\right)$ & $1+\lambda_{\min }\left(X_{k}\right)$ \\
\hline \hline 1 & $\{-0.9998,-0.2296,-0.0172,0.0030,0.2252,80.593\}$ & 0.0002 \\
\hline 2 & $\{-0.6573,-0.1834,-0.0031,0.0007,0.1882,52.519\}$ & 0.3427 \\
\hline 3 & $\{-0.4519,-0.1427,-0.0008,0.0026,0.16481,34.05\}$ & 0.5481 \\
\hline 4 & $\{-0.3221,-0.1040,-0.0016,0.0042,0.1449,21.364\}$ & 0.6779 \\
\hline 5 & $\{-0.2380,-0.0689,-0.0019,0.0040,0.12401,12.617\}$ & 0.7620 \\
\hline 6 & $\{-0.1812,-0.0407,-0.0018,0.0031,0.1000,6.7195\}$ & 0.8188 \\
\hline 7 & $\{-0.1425,-0.0215,-0.0012,0.0018,0.0713,2.9591\}$ & 0.8575 \\
\hline 8 & $\{-0.1296,-0.0117,-0.0002,0.0002,0.0367,0.852\}$ & 0.8704 \\
\hline 9 & $\{-0.2192,-0.01162,-0.0006,0.0006,0.0074,0.1055\}$ & 0.7808 \\
\hline 10 & $\{0,0,0,0,0,0\}$ & 1 \\
\hline
\end{tabular}

solution of (2.2) has the following explicit form:

$$
P_{0}=\left[\begin{array}{cccccc}
1.3097 & 0.35454 & 0.0033206 & 0.019828 & -0.25039 & -0.0064344 \\
0.35454 & 0.45562 & -0.047959 & 0.015024 & -0.12392 & -0.0035059 \\
0.0033206 & -0.047959 & 1.2772 & 0.13327 & -1.778 & -0.047567 \\
0.019828 & 0.015024 & 0.13327 & 0.092803 & -0.8661 & -0.031908 \\
-0.25039 & -0.12392 & -1.778 & -0.8661 & 20.29 & 0.61192 \\
-0.0064344 & -0.0035059 & -0.047567 & -0.031908 & 0.61192 & 0.086183
\end{array}\right] .
$$

The control feedback (2.1) can now be defined and has the following expression

$$
\begin{aligned}
& F_{* 0}=R^{-1} B^{T} P_{0}=\left[\begin{array}{cccccc}
4.4318 & 5.6952 & -0.59949 & 0.1878 & -1.5491 & -0.043824 \\
-0.13405 & -0.07304 & -0.99097 & -0.66474 & 12.748 & 1.7955
\end{array}\right], \\
& u=-F_{* 0} x
\end{aligned}
$$

Moreover, we have

$$
Q_{0}=\bar{Q}_{0}+F_{* 0}^{T} R F_{* 0} \quad \Rightarrow \quad \lambda_{\min }\left(Q_{0}\right)=\lambda_{\min }\left(\bar{Q}_{0}\right)=1 \quad\left(\lambda_{\min }\left(F_{* 0}^{T} R F_{* 0}\right)=0\right) .
$$

Note that Table 2 includes the computation of the spectra of matrices $X_{k}=\left(\bar{A}_{0}+\bar{A}_{1} \bar{\Delta}_{0}-\right.$ $\left.A_{q_{k}}\right)^{T} P_{0}+P_{0}\left(\bar{A}_{0}+\bar{A}_{1} \bar{\Delta}_{0}-A_{q_{k}}\right)$. Additionally we test the condition (2.8) for the values of $k \in$ $\{1, \ldots, 10\}$.

\subsection{Simulation Results}

This section includes the simulation results involving the proposed stabilizing feedback control. In Fig. 3 we present some simulation results when applying the LQ state feedback (3.23) to the given $S W Q A$ system represented by $(3.1)-(3.5)$. We have followed here the smooth take-off path (3.12) and (3.7) with $\bar{V}_{0}=1[\mathrm{~m} / \mathrm{s}], \bar{V}_{N}=15[\mathrm{~m} / \mathrm{s}]$ and $t_{N}=5[\mathrm{~m} / \mathrm{s}]$. Moreover, the state components are given as follows: $x=\left[\begin{array}{lll}x_{\mathbf{x}}^{T} & x_{\mathbf{z}}^{T} & x_{\theta}^{T}\end{array}\right]^{T}, x_{\mathbf{x}}=\left[\begin{array}{ll}\hat{\mathbf{x}} & \mathrm{d} \hat{\mathbf{x}} / \mathrm{d} t\end{array}\right]^{T}, x_{\mathbf{z}}=\left[\begin{array}{ll}\hat{\mathbf{z}} & \mathrm{d} \hat{\mathbf{z}} / \mathrm{d} t\end{array}\right]^{T}, x_{\theta}$ 
12 of 14

$=\left[\begin{array}{ll}\theta & \mathrm{d} \theta / \mathrm{d} t\end{array}\right]^{T}$, and the control actions, $f$ and $\tau_{q}$, are obtained from $(3.11),(3.13)$ and $(3.8)-$ $(3.11)$.

From the information presented on Fig. 3 we can conclude that the $S W Q A$ tracks correctly the smooth take-off path (3.12) and (3.7) and has a stable behavior.

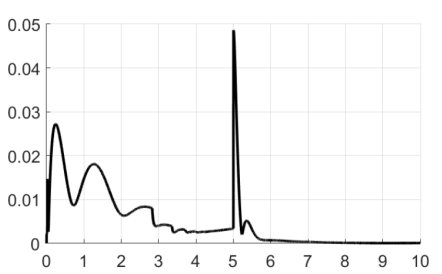

(a)

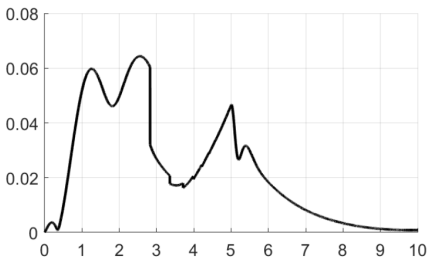

(b)

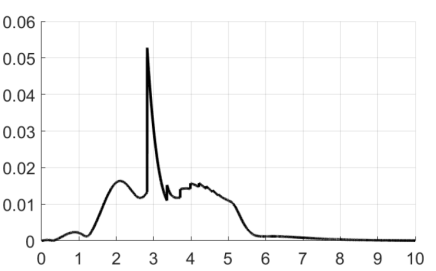

(c)

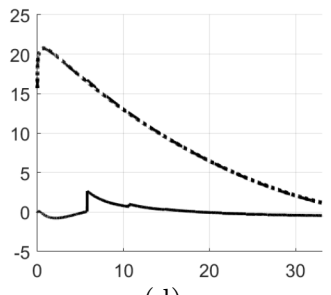

(d)

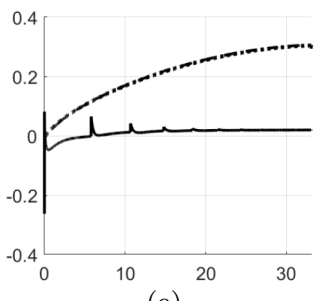

(e)

Fig. 3. Simulation results. (a) $\left\|x_{\theta}\right\|$. (b) $\left\|x_{\mathbf{Z}}\right\|$. (c) $\left\|x_{\mathbf{x}}\right\|$. (d) Dotted line: $\bar{F}$, dashed line: $F=\bar{F}+f$, solid line: $10 f$. (e) Dotted line: $\bar{T}_{q}$, dashed line: $T_{q}=\bar{T}_{q}+\tau_{q}$, solid line: $10 \tau_{q}$.

\section{CONCLUDING REMARKS}

In this paper, we have proposed a Riccati Equation based stabilizing state feedback for a wide class of switched dynamic models of the fluing objects type. The obtained matrices of the main state space representation (1.1) associated with the class of switched system possess a useful structure similar to (Narendra et al, 1994). Moreover, the admissible switching mechanisms have a generic hybrid nature studied in Azhmyakov (2019). These switched dynamic models make it possible to consider the useful formal state space representation (1.1)-(1.2), where the essential parameters of constant system structure is given by the simple model (1.2). Moreover, (1.5) are determined by the triplet $\left(\bar{A}_{0}, \bar{A}_{1}, \bar{\Delta}_{0}\right)$. Note that the combinatorial structure of the locations set in switched systems we examined is in fact represented by the matrix $\bar{D}\left(q_{i}\right)(c f$. $(1.5))$.

In our contribution, we have developed a kind of "robustness" result. This robustness is understood with respect to a possible (admissible) switching mechanism such that the Riccati Equation based stabilization feedback (2.1) and (2.2) stabilizes the initially given dynamic system (1.1)-(1.3). This stabilization is implemented under the assumption of an unknown dynamic location $q \in \mathscr{Q}$. We only assumed the knowledge of the essential parameters associated with the constant system structure (1.2) and (1.5) which was determined by the triple $\left(\bar{A}_{0}, \bar{A}_{1}, \bar{\Delta}_{0}\right)$.

The formal proof of Theorem 2.2 we presented involves some recent results from (Bonilla et al, 2015b). Finally note that the proposed Riccati Equation based stabilization feedback we 
developed not only guarantees the stability property of the Single Wing Quadrotor Aircraft in a concrete flying mode but also implies the stability during the mode change, namely, during the change from the Quadrotor Flight Envelope mode to the Airplane Flight Envelope.

Acknowledgement Ortiz Castillo thanks the french ambassy in México, that permitted a stay at 'Laboratoire des Sciences du Numérique de Nantes (LS2N)', France, during his master at 'Centro de Investigación y de Estudios Avanzados del Instituto Politécnico Nacional', México, in the framework of the program 'Discover Science in France'.

\section{References}

Azhmyakov, V. (2019). A Relaxation Approach to Optimal Control of Hybrid and Switched Systems, Elsevier, Oxford.

Bonilla, M., Malabre, M. and Azhmyakov, V. (2015a). An implicit systems characterization of a class of impulsive linear switched control processes. Part 1: Modeling. Nonlinear Analysis: Hybrid Systems, 15 , pp. 157-170.

Bonilla, M., Malabre, M. and Azhmyakov, V. (2015b). An implicit systems characterization of a class of impulsive linear switched control processes. Part 2: Control. Nonlinear Analysis: Hybrid Systems, 18, pp. 15-32.

Bonilla, M., Blas, L.A., Azhmyakov, V., Malabre, M. and Salazar, S. (2020). Robust Structural Feedback Linearization Based on the Nonlinearities Rejection. Journal of the Franklin Institute, 357, pp. 2232-2262.

Cook, M.V. (2013). Flight Dynamics Principles. A Linear Systems Approach to Aircraft Stability and Control, Elsevier, London.

Kailath, Th. (1980). Linear systems, Prentice-Hall, New Jersey.

Karcanias, N. and Livada, M. (2019). Complex Systems and Control: The Paradigms of Structure Evolving Systems and System of Systems. In: Zattoni E., Perdon A., Conte G. (eds) Structural Methods in the Study of Complex Systems. Lecture Notes in Control and Information Sciences, vol 482 Springer, pp. 3-55. ISBN 978-3-030-18571-8, ISBN 978-3-030-18572-5 (eBook).

Liberzon, D. (2003). Switching in Systems and Control, Springer Science, New York.

Narendra, K.S. and Balakrishnan, J. (1994). A common Lyapunov function for stable LTI systems with commuting A-matrices, 39(12), pp. 2469-2471. 
Ortiz Castillo, M.A., Bonilla, M., Loiseau, J.J., Malabre, M. and Azhmyakov, V. (2020). On the LQ Based Stabilization for a Class of Switched Dynamic Systems. Preprints of the $1^{\text {st }}$ Virtual IFAC World Congress IFAC-V 2020, 6 pp, Berlin, Deutschland, July 11-17, 2020.

Powers, C., Mellinger, D., and Kumar, V. (2014). Quadrotor Kinematics and Dynamics. In Chapter 16 of Handbook of unmanned aerial vehicles, editors Valavanis, K. P., \& Vachtsevanos, G. J. New York: Springer Publishing Company, Incorporated.

Selig M.S., C.A. Lyon, P. Gigure, C.N. Ninham, and J.J. Guglielmo. Summary of Low-Speed Airfoil Data, vol. 2, SoarTech Publications, Virginia Beach, VA, USA; 1996.

Stewart, G.W. (1973). Introduction to matrix computation. Academic Press, New York.

\section{Appendix}

5. Coefficients of $(3.15)$

$$
\begin{aligned}
& \stackrel{\circ}{a} \hat{\mathbf{x}} \mathbf{k} \approx\left(m \bar{V}\left(t_{k}\right)\right)^{-1}\left(-\stackrel{\circ}{D}_{V, k} \bar{V}\left(t_{k}\right)+\left(\stackrel{\circ}{D}_{\alpha, k}-L_{k}+\stackrel{\circ}{L}_{V, k} \bar{V}\left(t_{k}\right)\right) \overline{\boldsymbol{\alpha}}\left(t_{k}\right)\right),
\end{aligned}
$$

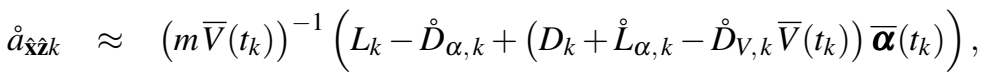

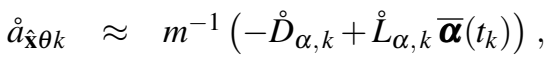

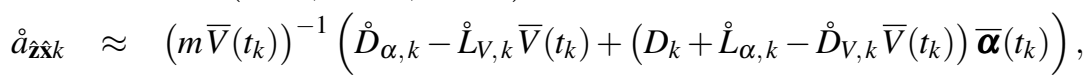

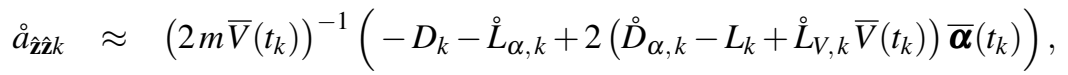

$$
\begin{aligned}
& \stackrel{\circ}{a}_{\hat{\mathbf{z}} \theta k} \approx-m^{-1}\left(\bar{F}\left(t_{k}\right)+\stackrel{\circ}{L}_{\alpha, k}+{\stackrel{\circ}{D_{\alpha, k}}} \overline{\boldsymbol{\alpha}}\left(t_{k}\right)\right) \text {, } \\
& \stackrel{\circ}{a}_{\theta \hat{\mathbf{x}} k} \approx \mathrm{I}_{y y}^{-1}\left(\stackrel{\circ}{V, k}_{V}+\overline{\bar{c}}\left(h-h_{0}\right) \stackrel{\circ}{L}_{V, k}\right)-\stackrel{\circ}{a}_{\theta \theta k} \overline{\boldsymbol{\alpha}}\left(t_{k}\right), \\
& \stackrel{\circ}{a}_{\theta \hat{\mathbf{z}} k} \approx \stackrel{\circ}{a}_{\theta \theta k}+\mathrm{I}_{y y}^{-1}\left(\stackrel{\circ}{M}_{V, k}+\overline{\bar{c}}\left(h-h_{0}\right) \stackrel{\circ}{L}_{V, k}\right) \overline{\boldsymbol{\alpha}}\left(t_{k}\right), \\
& \stackrel{\circ}{a}_{\theta \theta k}=\mathrm{I}_{y y}^{-1}\left(\stackrel{\circ}{\alpha, k}_{\alpha}+\overline{\bar{c}}\left(h-h_{0}\right) \stackrel{\circ}{L}, k\right) \text {, }
\end{aligned}
$$

where:

$$
\begin{array}{lll}
L_{k}=L\left(\bar{V}\left(t_{k}\right), \overline{\boldsymbol{\alpha}}\left(t_{k}\right)\right), & \stackrel{\circ}{L}_{\alpha, k}=\partial L\left(\bar{V}\left(t_{k}\right), \overline{\boldsymbol{\alpha}}\left(t_{k}\right)\right) / \partial \boldsymbol{\alpha}, & \stackrel{\circ}{L}_{V, k}=\partial L\left(\bar{V}\left(t_{k}\right), \overline{\boldsymbol{\alpha}}\left(t_{k}\right)\right) / \partial V, \\
D_{k}=D\left(\bar{V}\left(t_{k}\right), \overline{\boldsymbol{\alpha}}\left(t_{k}\right)\right), & \stackrel{\circ}{\alpha}, k_{\alpha,} \partial D\left(\bar{V}\left(t_{k}\right), \overline{\boldsymbol{\alpha}}\left(t_{k}\right)\right) / \partial \boldsymbol{\alpha}, & \stackrel{\circ}{D}, k_{V}=\partial D\left(\bar{V}\left(t_{k}\right), \overline{\boldsymbol{\alpha}}\left(t_{k}\right)\right) / \partial V, \\
M_{k}=M\left(\bar{V}\left(t_{k}\right), \overline{\boldsymbol{\alpha}}\left(t_{k}\right)\right), & \stackrel{\circ}{\alpha, k}_{\alpha, k}=\partial M\left(\bar{V}\left(t_{k}\right), \overline{\boldsymbol{\alpha}}\left(t_{k}\right)\right) / \partial \boldsymbol{\alpha}, & \stackrel{\circ}{M}, k_{V, k}=\partial M\left(\bar{V}\left(t_{k}\right), \overline{\boldsymbol{\alpha}}\left(t_{k}\right)\right) / \partial V .
\end{array}
$$

